13,09

\title{
Влияние перколяционного перехода на электропроводящие и оптические свойства сверхтонких металлических пленок
}

\author{
(C) О.А. Томилина, В.Н. Бержанский, С.В. Томилин \\ Физико-технический институт, Крымский федеральный университет им. В.И. Вернадского, \\ Симферополь, Россия \\ E-mail: olga_tomilina@mail.ru
}

Поступила в Редакцию 17 октября 2019 г.

В окончательной редакции 2 декабря 2019 г.

Принята к публикации 3 декабря 2019 г.

\begin{abstract}
Представлены результаты исследования особенностей изменения электрофизических, оптических и плазмонных свойств сверхтонких металлических пленок при перколяционном переходе от островковой структуры к сплошной. Показано, что при конденсации тонких пленок Тi и Pt изменение их электрической проводимости выше порога перколяции хорошо описывается в рамках классической перколяционной теории. Исследовано влияние перколяционного перехода в металлических пленках Аu на изменение типа плазмонного резонанса с поверхностного (распространяющиеся плазмон-поляритоны) на локализованный. Показано, что при переходе пленки в гранулированное состояние в окрестности перколяционного перехода наблюдается уменьшение добротности резонанса поверхностных плазмонова-поляритонов, связанное с возникновением условий для возбуждения локализованных плазмонов. Для всех исследованных покрытий определен порог перколяции.
\end{abstract}

Ключевые слова: сверхтонкая пленка, наноостровок, перколяционный переход, термоактивированная грануляция (ТАГ), электропроводность, спектр пропускания, плазмонный резонанс.

DOI: $10.21883 /$ FTT.2020.04.49129.610

\section{1. Введение}

Сверхтонкие и наноостровковые металлические пленки широко применяются для получения плазмонных и магнитоплазмонных сенсоров, для создания оптических систем, в устройствах фотовольтаических преобразователей, а также, в фотонике, спинтронике и т.д. [1-3]. Уникальность свойств наноструктурированных покрытий во многом определяется фононными и электронными процессами, протекающими как в объеме, так и на поверхности твердого тела. Известно, что при перколяционном переходе от островковой структуры пленки к сплошной и наоборот наблюдается существенное изменение физических свойств, связанных с пространственно-размерными ограничениями в электронной подсистеме покрытия [4-15]. Так, например в работах [4-13] в окрестности перколяционного пререхода наблюдались термо- и электрополевые эффекты, а также влияние оптического излучения на проводимость пленок. В работах $[14,15]$ наблюдалось изменение оптического поглощения с объемного на резонансное. Таким образом, исследование особенностей электропроводящих, оптических и плазмонных свойств тонких пленок при подобном структурном фазовом переходе является актуальной задачей.

\section{2. Методы получения и исследования образцов}

Синтез сверхтонких металлических пленок осуществлялся методом термического напыления в вакууме (остаточное давление не более $4 \cdot 10^{-4} \mathrm{~Pa}$ ) на диэлектрические подложки (ситалл - для исследования электрофизических свойств, гадолиний-галлиевый гранат GGG - для исследования оптических свойств, $\mathrm{SiO}_{2}$ - для исследования плазмонных свойств) при температуре $150^{\circ} \mathrm{C}$. Для формирования островковой структуры пленок применялся метод „тонкой заслонки“, принцип действия которого основан на формировании островкового покрытия непосредственно в процессе осаждения материала с малыми скоростями при условии реализации островкового механизма роста.

Суть метода состоит в механическом экранировании части потока напыляемого материала, при этом на подложке формируется зона геометрической ,полутени“, внутри которой пленка имеет градиент толщины (монотонный переход от однородного покрытия к чистой подложке). При этом в самой тонкой части градиента пленка имеет островковую структуру (размерный перколяционный переход). Более подробно схема метода „тонкой заслонки“ представлена в работе [16].

Исследования влияния перколяции на электрофизические свойства металлических пленок проводились на примере конденсации в вакууме сверхтонких пленок титана (Ti) и платины (Pt). Для этого на подложку через трафаретную маску предварительно напылялась контактная металлизация $\mathrm{Ag}$ к которой подключались электрические контакты. Измерение электропроводности тонких пленок осуществлялось в динамическом режиме (in suite) по двузондовой схеме при постоянном напряжении в слабых полях. 

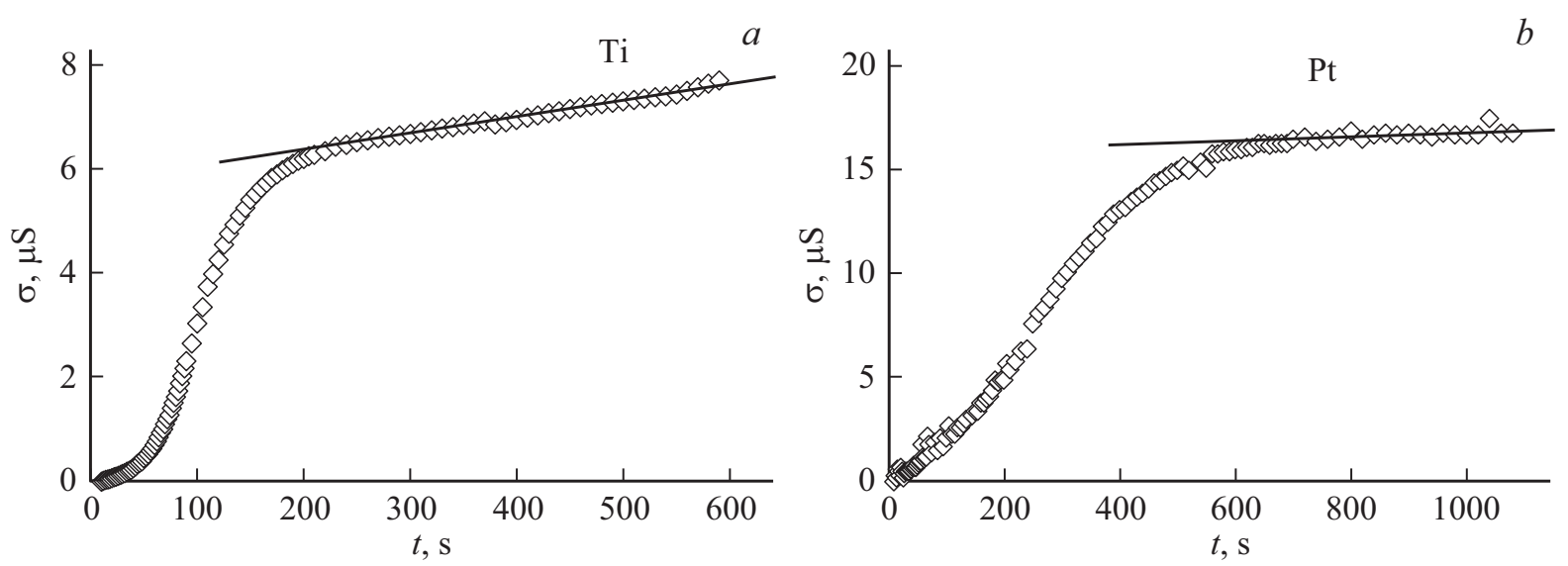

Рис. 1. Изменение проводимости пленок в процессе их конденсации: $a-\mathrm{Ti}, b-\mathrm{Pt}$. Точки - экспериментальные данные, линия - аппроксимация участка линейного роста проводимости.

Исследование оптических и плазмонных свойств тонких металлических пленок в окрестности перколяционного перехода проводилось на примере сверхтонких пленок золота $(\mathrm{Au})$. С этой целью методом „тонкой заслонки“ были синтезированы пленки золота с градиентом эффективной толщины $h_{\text {eff }}$ на подложках GGG и $\mathrm{SiO}_{2}$. При этом толщина пленки $\mathrm{Au} / \mathrm{GGG}$ в самой „толстой“ области составляла порядка $10 \mathrm{~nm}$, а пленки $\mathrm{Au} / \mathrm{SiO}_{2}-15 \mathrm{~nm}$, в самой тонкой части градиента толщина обоих пленок стремилась к $0 \mathrm{~nm}$. Именно в области сверхмалой толщины осуществлялся перколяционный переход в структуре пленок. Наличие островковой структуры фиксировалось методом растровой электронной микроскопии [17].

Для исследования спектров оптического пропускания пленки $\mathrm{Au} / \mathrm{GGG}$ с градиентом $h_{\text {eff }}$, был использован автоматизированный спектрофотометр КФК-З. Исследование спектров пропускания осуществлялось с шагом $l_{\text {step }}=0.5 \mathrm{~mm}$ вдоль градиента $h_{\text {eff. }}$.

Для исследования влияния перколяционного перехода на изменение свойств поверхностных плазмон-поляритонных состояний в пленке $\mathrm{Au} / \mathrm{SiO}_{2}$ с градиентом $h_{\text {eff }}$ использован метод возбуждения и регистрации поверхностного плазмонного резонанса (ППР) с использованием классической схемы Кретчмана [18].

\section{3. Результаты и их обсуждение}

\section{1. Проводимость сверхтонких пленок Тi и $\mathrm{Pt}$ В окрестности перколяционного перехода}

Результаты исследования временной динамики изменения проводимости пленок Ti и Pt при их конденсации представлены на рис. 1. При анализе кривых на рис. 1 мы принимаем допущение о квазипостоянной скорости осаждения покрытия (под скоростью осаждения следует понимать объем вещества, осаждаемый на единицу площади в единицу времени).
Как видно из рис. 1 на начальном этапе осаждения проводимость пленки чрезвычайно мала и увеличивается медленно, что обусловлено островковой структурой пленки ниже порога перколяции. Далее следует участок быстрого роста проводимости в результате „сращивания“ островков и формирования протяженных кластеров и перколяционных каналов с металлическим характером проводимости. На завершающем этапе наблюдается линейный рост проводимости со временем характерный для сплошного металлического покрытия (проводимость пропорционально увеличивается с ростом толщины пленки). Участок линейного роста на рис. 1 обозначен аппроксимирующей прямой. Как видно из рисунка, время достижения порога перколяции (выход на линейный участок) у Pt в 3 раза больше, чем у Тi. Это обусловлено тем, что $\mathrm{Pt}$ имеет более высокую температуру плавления и, как следствие, при прочих равных условиях испаряется с меньшей скоростью чем Ti, что и приводит к более низкой скорости напыления.

Анализ перколяционного перехода удобно рассматривать в зависимости не от времени осаждения $t$, a от доли занимаемой пленкой площади поверхности подложки $x[19,20]$ :

$$
\sigma=\sigma_{m}\left(x-x_{c}\right)^{\mu}
$$

где $\sigma_{m}-$ проводимость металлической фазы, $x_{c}-$ порог перколяции (доля поверхности, занимаемая пленкой, при которой начинается образование перколяционных каналов металлической проводимости), $\mu-$ критический индекс (для двумерной системы равен 1.3).

Для перехода от $t$ к $x$ примем следующие постулаты. Первый, в нулевой момент времени заполнение $x=0$ (чистая подложка). Второй, точка перехода проводимости на линейный участок соответствует заполнению $x=1$ (сплошная пленка). Третий, площадь занимаемой поверхности $x \sim t^{2 / 3}$. Последний постулат является следствием того, что при постоянной скорости 

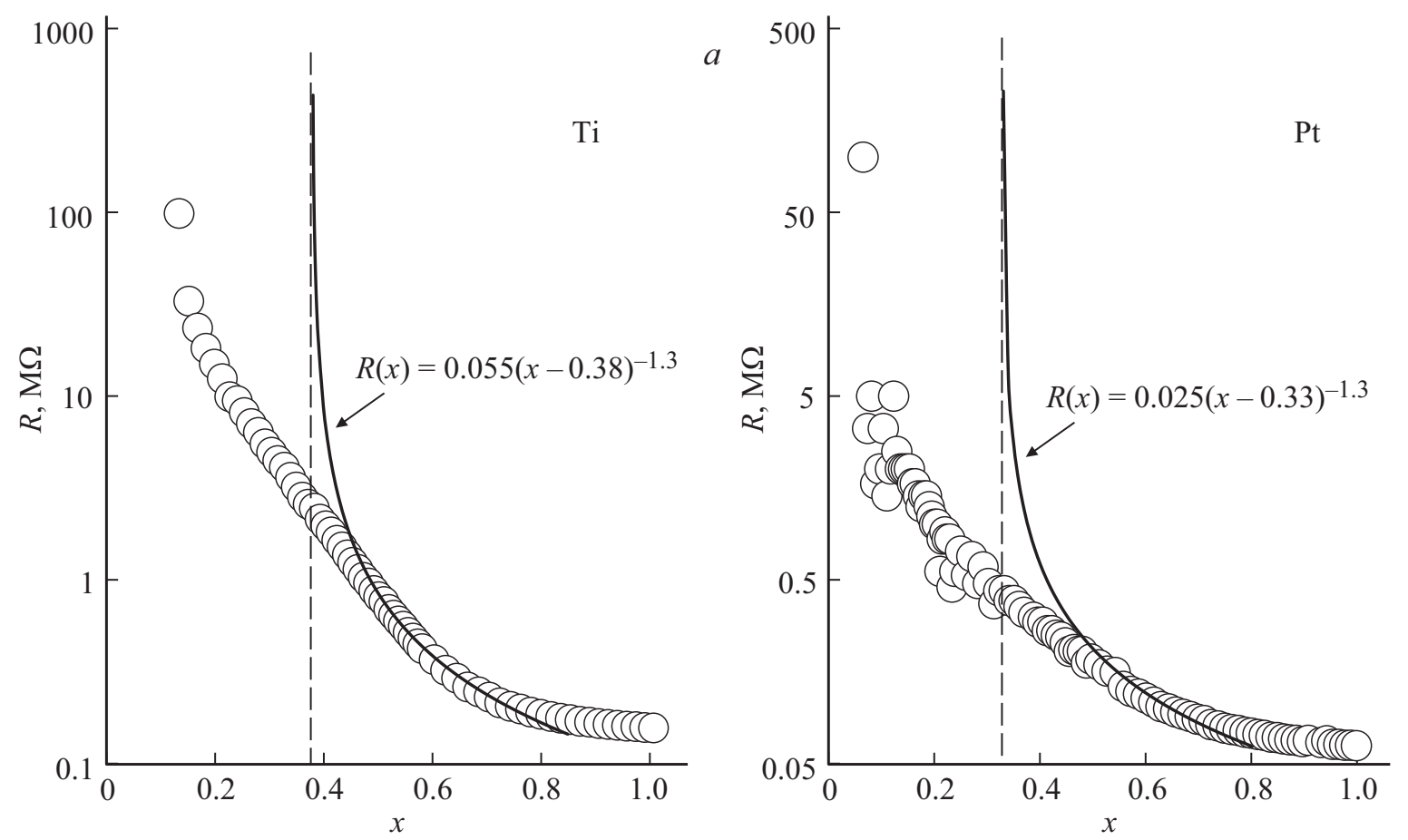

Рис. 2. Зависимость сопротивления пленок от поверхностной доли содержания металлической компоненты: $a-\mathrm{Ti}, b-\mathrm{Pt}$. Точки - экспериментальные данные, сплошные линии - их аппроксимация согласно уравнения (1), вид уравнения приведен на графиках.

объем осажденного металла пропорционален времени. Таким образом, для Ті получена функция перехода $x_{\mathrm{Ti}}=0.029 t^{2 / 3}$, а для $\mathrm{Pt}-x_{\mathrm{Pt}}=0.014 t^{2 / 3}$. Зависимость сопротивления пленок Тi и $\mathrm{Pt}$ от доли заполнения площади подложки представлена на рис. 2.

Анализ экспериментальных точек на основании уравнения (1) классической теории перколяции показан на рис. 2 сплошными линиями. На графиках приведены уравнения аппроксимирующих кривых и пунктиром показана горизонтальная асимптота, которая соответствует критической концентрации $x_{c}$ (порог перколяции). Для Ті значение $x_{c}=0.38$, для $\mathrm{Pt}-0.33$.

Из анализа графиков на рис. 2 видно, что выше порога перколяции экспериментальная зависимость $R(x)$ достаточно хорошо согласуется с классической перколяционной теорией. В то же время при $x<x_{c}$ экспериментальные данные расходятся с классической теорией, при этом сопротивление пленки имеет конечное значение вплоть до $x=0.1$. Отсутствие согласия эксперимента с классической теорией при $x<x_{c}$ может быть связано с квантовыми особенностями процессов переноса в островковой структуре пленок. Как показано нами в работах $[16,21]$ на примере островковых пленок $\mathrm{Fe}, \mathrm{Ni}$, Ti и Pt изменение проводимости пленки ниже порога перколяции определяется тепловыми и туннельными переходами электронов между отдельными островками и носит активационный характер. Полученные экспериментальные результаты также хорошо согласуются с результатами других авторов $[6,7,10,12,20]$.

\section{2. Оптические свойства сверхтонких пленок Au в окрестности перколяционного перехода. Локализованный плазмонный резонанс}

Результаты исследования изменения оптических свойств тонкой пленки $\mathrm{Au} / \mathrm{GGG}$ в различных точках градиента $h_{\text {eff }}$ представлены на рис. 3. Как видно из рисунка, для наиболее толстого участка пленки спектр пропускания соответствует спектру сплошной пленки $\mathrm{Au}$ [22].

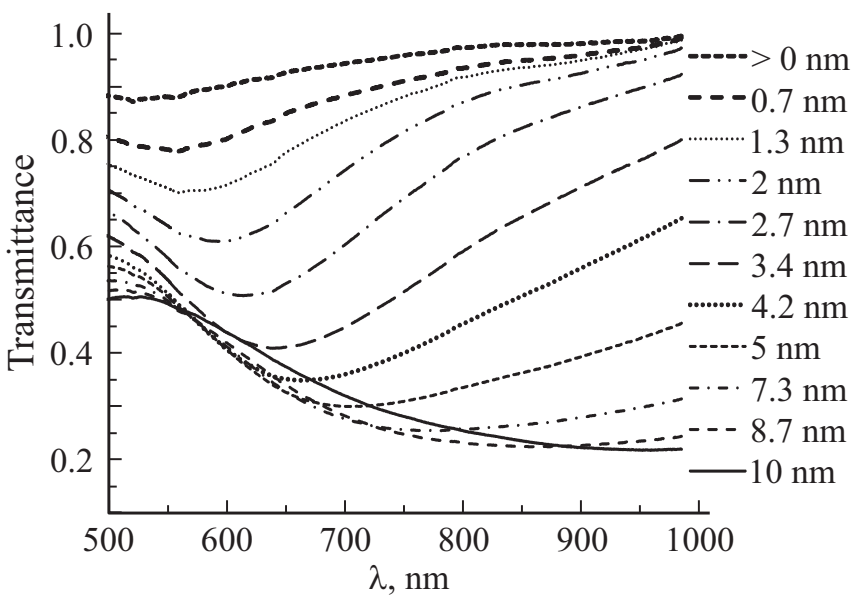

Рис. 3. Спектры пропускания пленки $\mathrm{Au} / \mathrm{GGG}$ с градиентом $h_{\text {eff }}$ в различных участках зоны „полутени“ (в легенде указано значение $h_{\mathrm{eff}}$. 


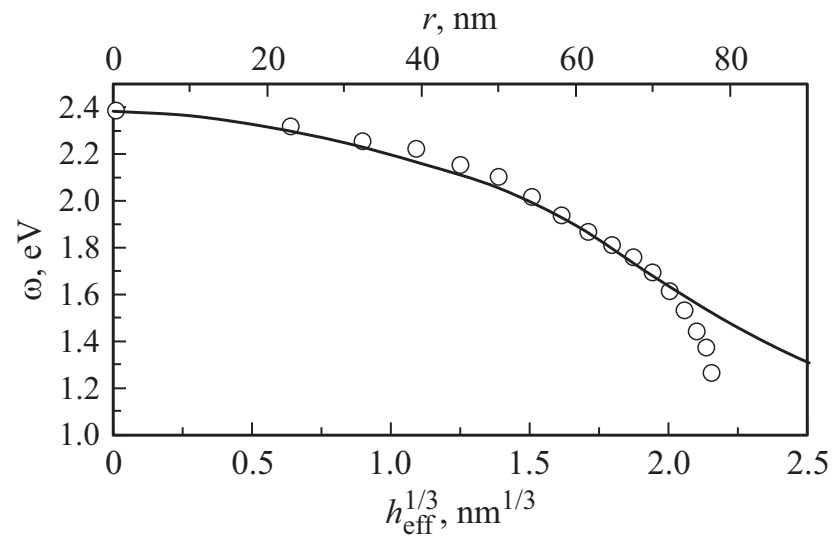

Рис. 4. Зависимость резонансной частоты $\omega$ локализованных плазмонов от $h_{\text {eff }}^{1 / 3}$, точки - экспериментальные данные, линия - теоретические результаты работы [23].

При уменьшении толщины пленки в спектрах пропускания появляется характерный минимум, обусловленный поглощением на локализованных плазмонах [22]. По мере уменьшения толщины покрытия положение плазмонного резонанса смещается в „синюю“ область спектра, а его добротность существенно возрастает, что свидетельствует о переходе структуры покрытия от сплошной к островковой (перколяционный переход).

Таким образом, локализованный плазмонный резонанс (ЛПР), наблюдаемый в виде спектрального минимума на резонансных частотах, может служить способом оценки особенностей перколяционного оптического перехода в сверхтонких металлических пленках.

Учитывая, что $h_{\text {eff }}$ по определению есть объем материала, осаждаемого на единичную площадь поверхности, можно предположить, что для островковой пленки наиболее вероятный радиус островка $r \sim h_{\text {eff }}^{1 / 3}$. На рис. 4 представлена зависимость резонансной частоты $\omega$ локализованных плазмонов (см. рис. 3) от $h_{\mathrm{eff}}^{1 / 3}$ (радиуса островка, выраженного в условных единицах). Данный вид экспериментальной кривой достаточно хорошо согласуется с теоретическими результатами работы [23] для дипольного резонанса (сплошная линия на графике). Рассогласование эксперимента с теорией для $r>70 \mathrm{~nm}$ обусловлено тем, что при диффузном механизме конденсации пленки островки преимущественно растут в плоскости, что приводит к нарушению соотношения $r \sim h_{\mathrm{eff}}^{1 / 3}$ при больших толщинах.

Для анализа „оптического“ перколяционного перехода в тонкой пленке $\mathrm{Au} / \mathrm{GGG}$ вдоль градиента $h_{\mathrm{eff}}$ с точки зрения классической теории перколяции рассмотрим изменение коэффициента экстинкции $E x t=1-T$, где $T$ - коэффициент пропускания. Очевидно, что экстинкция (поглощение и рассеяние падающего излучения) вне плазмонного резонанса будет в значительной мере определяться проводимостью металлического кластера, которая в свою очередь будет зависеть от размеров данного кластера. На рис. 5, $а$ представлена зависимость коэффициента экстинкции $E x t$ от эффективной толщины пленки $h_{\text {eff }}$ для значения длины волны $\lambda=985 \mathrm{~nm}$. Выбор данной длины волны сделан из необходимости максимального удаления от частот плазмонного резонанса.

Как видно из рис. 5, $a$ для малых эффективных толщин пленки характерна слабая, почти нулевая, экстинкция света, так как пленка является островковой и состоит преимущественно из мелких островков с узкой резонансной линией поглощения, при этом рассеяние на частицах, имеющих размер на порядок меньше длины волны, мало эффективно. Для толщин более $2 \mathrm{~nm}$ эффективность экстинкции резко возрастает и при достижении эффективной толщины пленки $h_{\mathrm{eff}}=10 \mathrm{~nm}$ переходит в объемное поглощение и отражение на сплошной пленке Au.
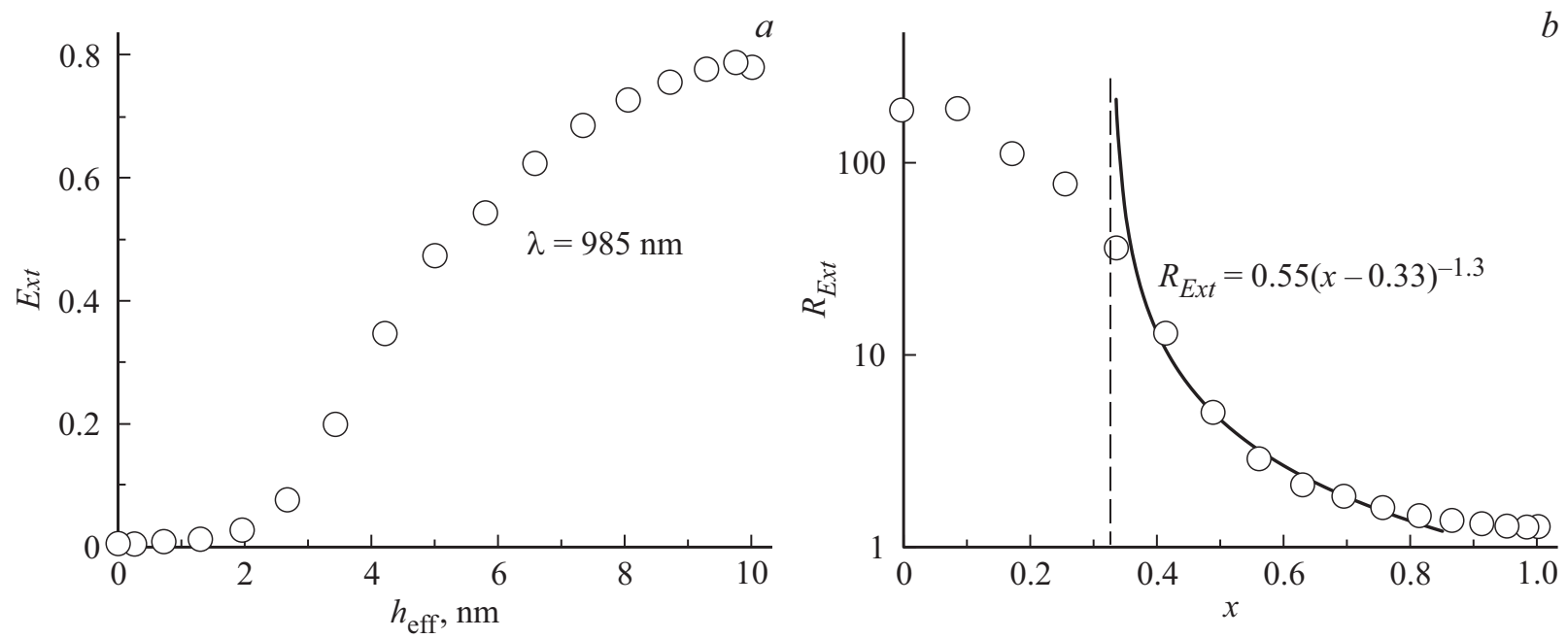

Рис. 5. Изменение коэффициента экстинкции на длине волны $985 \mathrm{~nm}$ пленки $\mathrm{Au} / \mathrm{GGG}$ вдоль градиента $h_{\mathrm{eff}}(a)$, зависимость величины $R_{E x t}=1 / E x t$ от степени заполнения подложки $x(b)$. Точки - экспериментальные данные, сплошная линия аппроксимация на основании уравнения (1), вид уравнения приведен на графиках. 

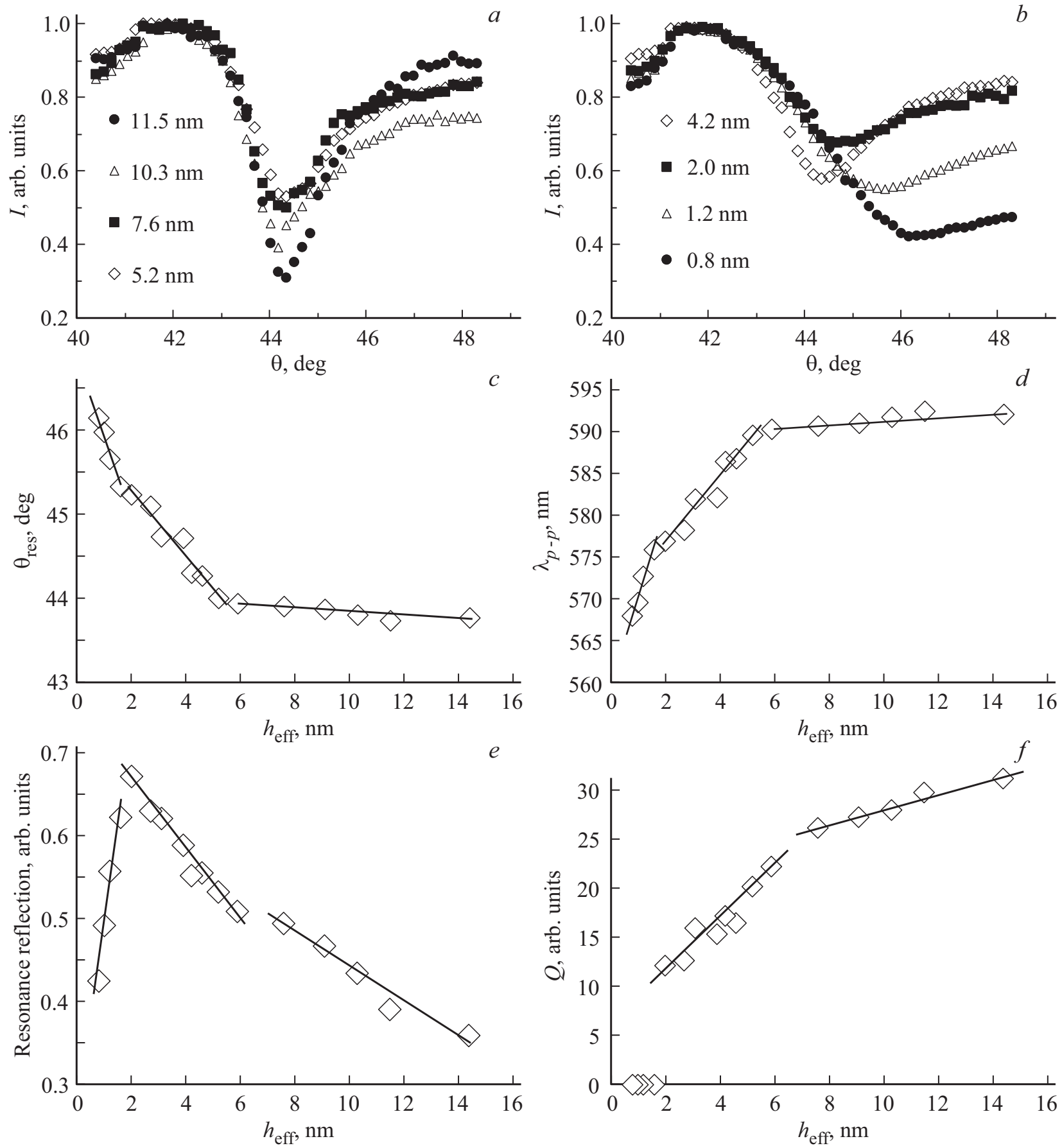

Рис. 6. Резонансные кривые ПППР в пленке $\mathrm{Au} / \mathrm{SiO}_{2}$ на различных участках вдоль градиента $h_{\text {eff }}(a, b)\left(\right.$ в легенде - значение $\left.h_{\mathrm{eff}}\right)$. Зависимость от $h_{\text {eff }}$ резонансного угла ПППР $(c)$, резонансной длины плазмон-поляритонной волны $(d)$, коэффициента отражения при условии резонанса (e), показателя добротности резонансных пиков ПППР $(\mathbf{e})$.

Зависимость на рис. 5, $a$ имеет вид характерный для классического перколяционного перехода, поэтому целесообразно провести анализ данного „оптического“ перехода по аналогии с электропроводящими свойствами на основании уравнения (1). Для перехода от эффективной толщины $h_{\mathrm{eff}}$ к степени заполнения подложки $x$ воспользуемся постулатами, описанными в подразделе 3.1. Так при $h_{\mathrm{eff}}=0$ степень заполнения $x=0$, а при $h_{\mathrm{eff}}=10 \mathrm{~nm}, x=1$. Связь между эффективной толщиной и площадью заполнения поверхности $x \sim h_{\mathrm{eff}}^{2 / 3}$. Таким образом для зависимости на рис. 5, a уравнение перехода имеет вид $x=0.2154 h_{\mathrm{eff}}^{2 / 3}$. В качестве аналога электрического сопротивления в данном случае будет выступать величина обратно пропорциональная коэффициенту экстинкции („сопротивление“ экстинкции, $\left.R_{E x t}=1 / E x t\right)$. Зависи- 
мость $R_{E x t}$ от степени заполнения х представлена на рис. $5, b$.

Как видно из представленного на рис. $5, b$ зависимость $R_{E x t}(x)$ достаточно хорошо описывается теоретическим уравнением (1) со значением порога перколяции $x_{c}=0.33$ (на графике аппроксимация показана сплошной линией), что соответствует $h_{\mathrm{eff}}=1.95 \mathrm{~nm}$.

Таким образом видно, что перколяционный переход в тонких пленках можно наблюдать по изменению оптических свойств двумя способами. Первый способ основан на анализе спектральных характеристик коэффициента пропускания (экстинкции) и регистрации возбуждения резонанса локализованных плазмонов. Второй заключается в анализе изменения коэффициента экстинкции на длинах волн, исключающих возбуждение резонансных плазмонов. Во втором случае „оптический“ перколяционный переход аналогичен „электрическому“ переходу и может быть описан в рамках классической теории.

\section{3. Плазмонные свойства сверхтонких пленок Au в окрестности перколяционного перехода. Поверхностный плазмонный резонанс}

Результаты исследования изменения резонансных свойств поверхностных плазмон-поляритонов в тонкой пленке $\mathrm{Au} / \mathrm{SiO}_{2}$ с градиентом $h_{\text {eff }}$ показаны на рис. $6, a, b$. Представлены нормированные резонансные кривые зависимости интенсивности отраженного луча $\lambda=632.8 \mathrm{~nm}$ от угла падения (в легенде указано значение $h_{\text {eff }}$ в точке исследования). Видно, что на рефлектометрических кривых наблюдается пик поверхностного плазмон-поляритонного резонанса (ПППР) [24]. При этом на самых тонких участках пленки Аu наблюдается „размытие“ резонансного пика, что свидетельствует о переходе к островковой структуре [25].

На рис. 6, $c-f$ представлены зависимости основных параметров ПППР от эффективной толщины $h_{\text {eff, та- }}$ ких как: резонансный угол $\theta_{\text {res }}\left(h_{\text {eff }}\right)$, длина резонансной плазмон-поляритонной волны $\lambda_{p-p}\left(h_{\mathrm{eff}}\right)$, интенсивность отраженного луча при условии резонанса $R\left(h_{\mathrm{eff}}\right)$ и показатель добротности резонансного пика $Q\left(h_{\mathrm{eff}}\right)$. Резонансная длина волны определяется из условия фазового синхронизма $\lambda_{p-p}=\lambda /\left(n \cdot \sin \theta_{\text {res }}\right)$, где $n=1.514-$ показатель преломления подложки $\mathrm{SiO}_{2}$. Показатель добротности ПППР может быть представлен как $k / \Delta k$, где $k$ - волновое число резонансной плазмон-поляритонной волны, а $\Delta k-$ разброс волновых чисел на полувысоте резонансного пика (ширина резонансной линии).

Следует обратить внимание, что совокупности точек на графиках (рис. 6,c-f) можно условно разбить на три группы с характером зависимости близким к линейному. Очевидно, что наличие подобных групп точек обусловлено структурными особенностями покрытия, в котором возбуждаются поверхностные плазмон-поляритонные (ПП) волны.
Так на участке 7-14.5 nm пленка имеет относительно высокую структурную однородность и сплошность, что подтверждается общим видом сравнительно четких резонансных пиков. Резонансный угол и, соответственно, длина волны резонансного плазмон-поляритона меняется незначительно (в пределах 1\%). Однако добротность и высота ПППР падает с уменьшением толщины пленки $\mathrm{Au}$, что связано с влиянием морфологии поверхности пленки и интерфейса ,пленка-подложка“ на распространение ПП волн при уменьшении толщины пленки Au.

В диапазоне эффективных толщин 2-6 nm величина резонансного угла существенно увеличивается (длина волны ПППР уменьшается) с уменьшением $h_{\text {eff. При }}$ этом характер изменения высоты резонансного пика и его добротности при уменьшении $h_{\mathrm{eff}}$ аналогичен предыдущему участку, отличается лишь коэффициент пропорциональности. Подобные отличия связаны со структурными изменениями в пленке и свидетельствуют о наличии в ней нанонеоднородностей.

При значениях $h_{\text {eff }}$ менее $2 \mathrm{~nm}$, структура пленки является наноостровковой, что подтверждается видом резонансных кривых ПППР и отражается на свойствах резонанса. В данном случае видно, что с уменьшением $h_{\text {eff }}$ резонансный угол резко возрастает и соответственно $\lambda_{p-p}$ уменьшается.

Особого внимания при рассмотрении заслуживает зависимость резонансного коэффициента отражения $R$ (Resonance reflection) от эффективной толщины пленки $\mathrm{Au} / \mathrm{SiO}_{2}$ (рис. 6,e). Она так же имеет 3 четко выраженных линейных участка. Минимальные значения $R$ наблюдается на „толстых“ участках градиентной пленки $\mathrm{Au} / \mathrm{SiO}_{2}$, для которых характерно малое затухание и высокая добротность ПП волн.

Резкий рост $R$ в диапазоне толщин 6-2 nm вызван уменьшением эффективности возбуждения светом бегущих ПП волн, что обусловлено началом спонтанной грануляции пленки. По этой же причине происходит и спад добротности ПППР.

В диапазоне толщин менее $2 \mathrm{~nm}$ наблюдается резкий спад коэффициента отражения, что свидетельствует об эффективном взаимодействия света с полностью гранулированной островковой структурой пленки (рассеяние на крупных кластерах и возбуждение локализованных плазмонных резонансов в наночастицах). Добротность ПППР для данного участка определить невозможно (условно принята за 0) из-за сильного размытия резонансного пика. Очевидно, что возбуждение ЛПР (локализованных плазмонов) на отдельных „изолированных“ островках будет тем эффективнее, чем выше степень грануляции пленки. Таким образом коэффициент отражения будет уменьшаться с толщиной, так как вероятность образования изолированных островков с уменьшением $h_{\text {eff }}$ будет увеличиваться.

Таким образом видно, что для градиентной пленки $\mathrm{Au} / \mathrm{SiO}_{2}$, перколяционный переход, как и в пленках $\mathrm{Au} / \mathrm{GGG}$, наблюдается в районе эффективной толщины 
$h_{\text {eff }}=2 \mathrm{~nm}$, что наглядно отображается на виде угловых спектров ПППР.

\section{4. Заключение}

Таким образом, в работе показано, что при перколяционном переходе в сверхтонких металлических пленках наблюдается существенное изменение физических свойств, обусловленное размерным ограничением электронной подсистемы твердого тела.

Исследование in-suite динамики изменения проводимости в процессе осаждения пленок Ті и Pt в окрестности порога перколяции при $x>x_{c}$ показало хорошее согласование с классической перколяционной теорией: при значении критического индекса $\mu=1.3$, порог перколяции близок к теоретическому значению и составляет $x_{c}=0.38$ для Ті и $x_{c}=0.33$ для Pt. Отсутствие согласования экспериментальных данных с теорией при $x<x_{c}$ связано с квантовыми особенностями процессов переноса носителей заряда в островковой структуре пленок.

При перколяционном переходе в спектрах пропускания градиентной наноразмерной пленки Au/GGG ниже порога перколяции наблюдается образование спектральных минимумов, обусловленное резонансным возбуждением локализованных плазмонов. При увеличении размера островков ЛПР испытывает „красное“ смещение, а при $x>x_{c}$ образование крупных кластеров приводит к существенному уширению и размытию спектра ЛПР. Экспериментально выявленное изменение спектрального положения плазмонного резонанса при изменении размера наночастиц хорошо согласуется с теоретическими данными.

Зависимость коэффициента экстинкции света вне плазмонного резонанса от степени грануляции пленок $\mathrm{Au} / \mathrm{GGG}$ описывается соответствующими выражениями теории перколяции. Определенное таким образом для пленки $\mathrm{Au} / \mathrm{GGG}$ значение порога перколяции также близко к теоретическому $x_{c}=0.33$, что соответствует эффективной толщине покрытия $h_{\mathrm{eff}}=1.95 \mathrm{~nm}$.

Исследованы условия резонансного возбуждения поверхностных (бегущих) плазмон-поляритонов в градиентной пленке $\mathrm{Au} / \mathrm{SiO}_{2}$ в окрестности перколяционного перехода. Показано существенное влияние грануляции пленки на эффективность возбуждения ПППР. Ниже порога перколяции наблюдается сильное „размытие“ резонансного пика, при этом эффективность поглощения излучения возрастает, что связано с резонансным возбуждением локализованных плазмонов в металлических наночастицах. Перколяционный переход в пленке $\mathrm{Au} / \mathrm{SiO}_{2}$ наблюдается при толщине $h_{\mathrm{eff}}=2 \mathrm{~nm}$.

\section{Финансирование работы}

Работа выполнена при финансовой поддержке гранта РНФ № 19-72-20154.

\section{Конфликт интересов}

Авторы заявляют, что у них нет конфликта интересов.

\section{Список литературы}

[1] Е.В. Костюкевич, С.А. Костюкевич. Оптоэлектроника и полупроводниковая техника 49, 60 (2014).

[2] В.В. Климов. УФН 178, 8, 875 (2008).

[3] В.И. Балюба, В.Ю. Грицык, Т.А. Давыдова, В.М. Калыгина, С.С. Назаров, Л.С. Хлудкова. ФТП 39, 2, 285 (2005).

[4] I.A. Gladskikh, N.B. Leonov, S.G. Przhibel'skii, T.A. Vartanyan. Nanosystems: Phys., Chem., Mathem. 4, 4, 524 (2013).

[5] Т.А. Вартанян, И.А. Гладских, Н.Б. Леонов, С.Г. Пржибельский. ФТТ 56, 4, 783 (2014).

[6] И.В. Антонец, Л.Н. Котов, С.В. Некипелов, Е.А. Голубев. ЖТФ 74, 3, 24 (2004).

[7] И.В. Антонец, Л.Н. Котов, С.В. Некипелов, Е.Н. Карпушов. ЖТФ 74, 11, 1022004.

[8] А.П. Болтаев, Ф.А. Пудонин. ЖЭТФ 13, 3(9), 500 (2006).

[9] В.Б. Лобода, С.Н. Хурсенко. ЖЭТФ 5, 11, 911 (2006).

[10] J.S. Agustsson, U.B. Arnalds, A.S. Ingason, K.B. Gylfason, K. Johnsen, S. Olafsson, J.T. Gudmundsson. J. Phys. Conf. Ser. 100, 1 (2008).

[11] И.А. Гладских, В.А. Полищук, Т.А. Вартанян. ФТТ 59, 3, 582 (2017).

[12] А.П. Болтаев, Н.А. Пенин, А.О. Погосов, Ф.А. Пудонин. ЖЭТФ 4, 954 (2004)

[13] А.П. Болтаев, Н.А. Пенин, А.О. Погосов, Ф.А. Пудонин. ЖЭТФ 123, 5, 1072 (2003).

[14] K. Uchida, H. Adachi, D. Kikuchi, S. Ito, Z. Qiu, S. Maekawa, E. Saitoh. Nature Commun. 65910 (2015). http://www.nature.com/articles/ncomms6910.

[15] A. Axelevitch, B. Apter, G. Golan. Opt. Expr. 21, 4, 4126 (2013).

[16] С.В. Томилин, В.Н. Бержанский, А.С. Яновский, О.А. Томилина. Поверхность. Рентгеновские синхротронные и нейтронные исследования 8, 96 (2016).

[17] S.V. Tomilin, V.N. Berzhansky, A.N. Shaposhnikov. J. Phys: Conf. Ser. 741, 012113 (2016).

[18] С.А. Майер. Плазмоника: теория и приложения / Пер. с англ. Т.С. Нечаевой, Ю.В. Колесниченко / Под ред. С.С. Савинского. НИЦ „Регулярная и хаотическая динамика“. М.Ижевск 2011. 296 с.

[19] Ю.Ю. Тарасевич. Перколяция: теория, приложения, алгоритмы. Едиториал УРСС, М. (2002). 112 с.

[20] В.Ф. Гантмахер. Электроны в неупорядоченных средах. ФИЗМАТЛИТ, М. (2005). 232 с.

[21] С.В. Томилин, В.Н. Бержанский, Е.Т. Милюкова, О.А. Томилина, А.С. Яновский. ФТТ 60, 7, 1251 (2018).

[22] B.-P. Zhang, H. Masumoto, Y. Someno, T. Goto. Mater. Transact. 44, 2, 215 (2003).

[23] K. Kolwas, A. Derkachova. Opto-Electr. Rev. 18, 4, 421 (2010).

[24] J. Guo, Zh. Zhu, W. Deng. Appl. Opt. 38, 31, 6550 (1999).

[25] L.L. Frumin, A.V. Nemykin, S.V. Perminov, D.A. Shapiro. J. Opt. 15, 8, 085002 (2013).

Редактор К.В. Емцев 